Journal for ImmunoTherapy of Cancer

\title{
Tisagenlecleucel infusion in patients with relapsed/refractory ALL and concurrent serious infection
}

\author{
Erin M Hall, Dwight E Yin, Rakesh K Goyal, Atif A Ahmed, Grace S Mitchell, \\ Shawn D St. Peter, Terrie G Flatt, Ibrahim A Ahmed, Weijie Li, \\ Richard J Hendrickson, Keith J August, G Doug Myers
}

To cite: Hall EM, Yin DE, Goyal RK, et al. Tisagenlecleucel infusion in patients with relapsed/refractory ALL and concurrent serious infection. Journal for ImmunoTherapy of Cancer 2021;9:e001225. doi:10.1136/jitc-2020-001225

- Additional material is published online only. To view, please visit the journal online (http://dx.doi.org/10.1136/jitc2020-001225).

Accepted 06 December 2020

Check for updates

(C) Author(s) (or their employer(s)) 2021. Re-use permitted under CC BY-NC. No commercial re-use. See rights and permissions. Published by BMJ.

School of Medicine, University of Missouri Kansas City, Children's Mercy Kansas City, Kansas City, Missouri, USA

Correspondence to

Dr G Doug Myers;

gdmyers@cmh.edu

\section{ABSTRACT}

Background Tisagenlecleucel, an anti-CD19 chimeric antigen receptor T (CAR-T) cell therapy, has demonstrated durable efficacy and a manageable safety profile in pediatric and young adult patients with relapsed/refractory B-cell acute lymphoblastic leukemia (B-ALL) in the ELIANA pivotal trial and real-world experience. Experience from investigator-led studies prior to ELIANA suggests that infections and inflammatory conditions may exacerbate the severity of cytokine release syndrome (CRS) associated with CAR-T cell therapy, leading to extreme caution and strong restrictions for on-study and commercial infusion of tisagenlecleucel in patients with active infection. CRS intervention with interleukin (IL)-6 blockade and/ or steroid therapy was introduced late in the course during clinical trials due to concern for potential negative effect on efficacy and persistence. However, earlier CRS intervention is now viewed more favorably. Earlier intervention and consistency in management between providers may promote broader use of tisagenlecleucel, including potential curative therapy in patients who require remission and recovery of hematopoiesis for management of severe infection.

Main body Patient 1 was diagnosed with B-ALL at 23 years old. Fourteen days before tisagenlecleucel infusion, the patient developed fever and neutropenia and was diagnosed with invasive Mucorales infection and BK virus hemorrhagic cystitis. Aggressive measures were instituted to control infection and to manage prolonged cytopenias during CAR-T cell manufacturing. Adverse events, including CRS, were manageable despite elevated inflammatory markers and active infection. The patient attained remission and recovered hematopoiesis, and infections resolved. The patient remains in remission $\geq 1$ year postinfusion.

Patient 2 was diagnosed with pre-B-ALL at preschool age. She developed severe septic shock 3 days postinitiation of lymphodepleting chemotherapy. After receiving tisagenlecleucel, she experienced CRS with cardiac dysfunction and extensive lymphadenopathy leading to renovascular compromise. The patient attained remission and was discharged in good condition to her country of origin. She remained in remission but expired on day 208 postinfusion due to cardiac arrest of unclear etiology. Conclusions Infusion was feasible, and toxicity related to tisagenlecleucel was manageable despite active infections and concurrent inflammation, allowing attainment of remission in otherwise refractory pediatric/young adult ALL. This may lead to consideration of tisagenlecleucel as a potential curative therapy in patients with managed active infections.

\section{BACKGROUND}

Anti-CD19 chimeric antigen receptor $\mathrm{T}$ (CAR-T) cell therapy (tisagenlecleucel) has demonstrated durable efficacy and manageable safety for pediatric and young adult patients with relapsed or refractory $(\mathrm{r} / \mathrm{r})$ B-cell acute lymphoblastic leukemia (B-ALL) ${ }^{1-3}$ In the single-arm, phase 2 ELIANA study (NCT02435849), 79 patients with $\mathrm{r} / \mathrm{r}$ B-ALL (age range, 3-24 years) were infused with a single dose of tisagenlecleucel. The overall remission rate (complete remission and complete remission with incomplete blood count recovery) was $82 \%$ within 3 months. Adverse events of special interest included cytokine release syndrome (CRS, 77\%) and neurological toxicities $(39 \%) .{ }^{3}$ Patients with active infections were not enrolled and infusion was held for cases of uncontrolled infection or inflammation. Current US prescribing information warns against administration of tisagenlecleucel to patients with active infection or inflammatory conditions. ${ }^{1}$ However, there remains a lack of data for CAR-T cell therapy in patients with active, managed infections. Furthermore, there are varying levels of comfort in treating patients with different levels of disease burden, infection types, and comorbidities. The publication of clinician and patient experiences with difficult cases should benefit providers treating patients in need of potentially curative therapy but who may be delayed or denied therapy based on prior guidelines for infusion. Herein, we describe two patients with $\mathrm{r} / \mathrm{r}$ B-ALL and concurrent infection and/or organ dysfunction who were safely infused with and responded to tisagenlecleucel. 

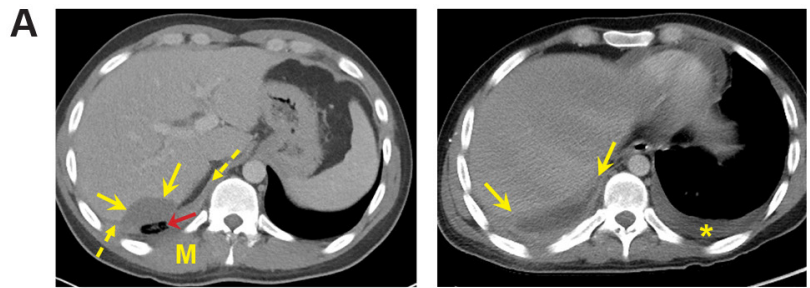

B
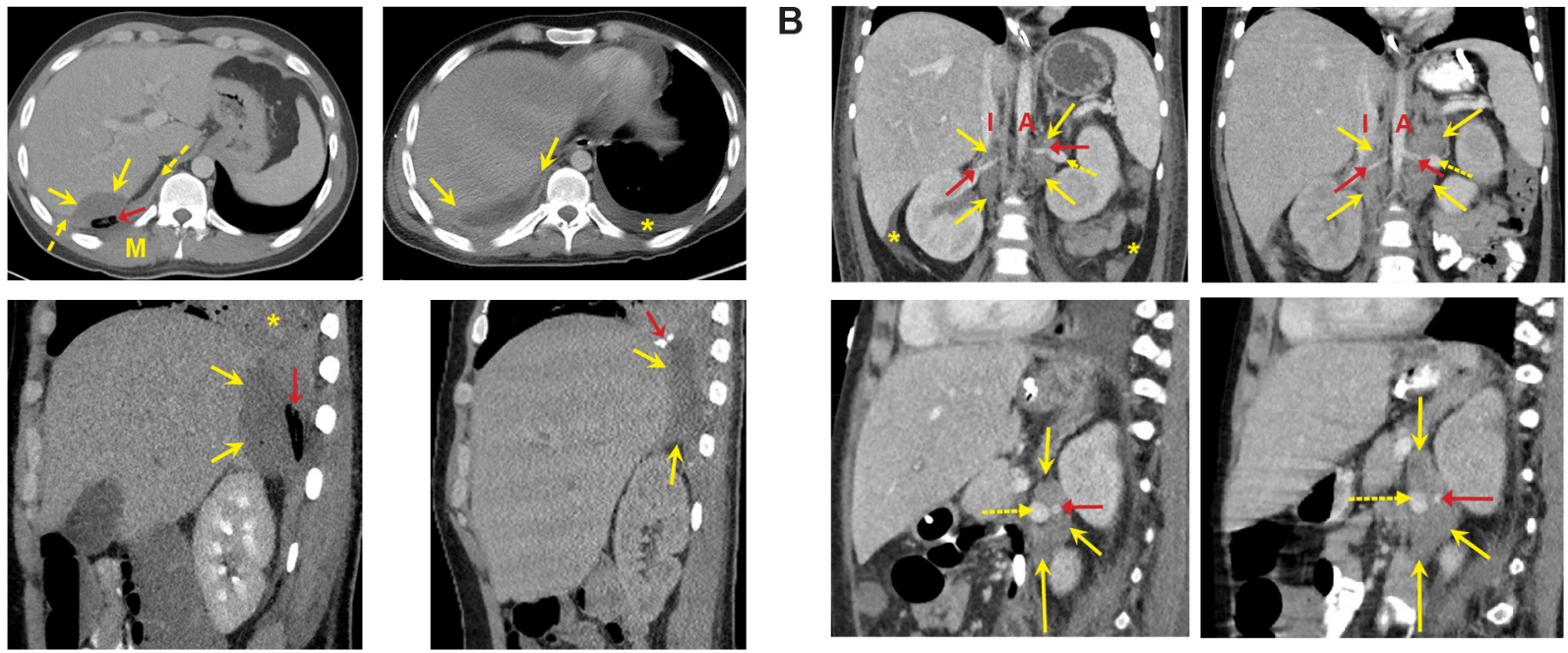

Figure 1 Contrast-enhanced CT scans for both patients before tisagenlecleucel and after tisagenlecleucel infusion. (A) Patient 1: CT scans performed at diagnosis and following treatment. Axial (top left) and sagittal (bottom left) images at diagnosis demonstrate a lenticular hypodense structure (yellow arrows) centered at, and Inseparable from, the abnormally thickened right diaphragm (dashed arrows). This causes invasion or indentation of the adjacent posterior right hepatic contour. A small tubular air-filled component is present within the diaphragm (red arrows). Subtle asymmetric enlargement of the right $\mathrm{M}$ raises the question of chest wall involvement. There is consolidation of the adjacent right lung (*). Post-treatment axial (top right) and sagittal (bottom right) images demonstrate resolution of the hypodense structure, with a residual thick-walled enhancing fluid collection along the diaphragm (yellow arrows). Note improved contour of the posterior right liver. Anastomotic sutures (red arrow) and a small left pleural effusion $\left(^{*}\right)$ are present. (B) Patient 2: CT scans performed at diagnosis and following treatment. Coronal (top left) and sagittal (bottom left) images prior to treatment demonstrate mildly enhancing soft tissue nodal masses (large yellow arrows) encircling the renal arteries (red arrows) and veins (small dashed arrows). A and I are partially visualized. A small amount of free fluid $\left(^{*}\right)$ is present. Post-treatment coronal (top right) and sagittal (bottom right) images demonstrate enlargement of the soft tissue nodal masses (large yellow arrows) encircling the renal arteries (red arrows) and veins (small dashed arrows). A and I are partially visualized. A, aorta; I, intrahepatic inferior vena cava; M, paraspinal muscle.

\section{Case presentation}

Patient 1

A 23-year-old male was diagnosed with B-ALL 18 months before tisagenlecleucel infusion. His disease relapsed after standard and salvage chemotherapy with development of refractory disease following haploidentical hematopoietic stem cell transplant. An additional text file includes further treatment history (see online supplemental file). The patient was evaluated for tisagenlecleucel and leukapheresis occurred 17 months after diagnosis. Bone marrow evaluation after bridging chemotherapy revealed $95 \%$ blasts in a normocellular marrow.

Fourteen days preinfusion, the patient was admitted for fever/neutropenia. Due to persistent fevers, a CT scan was obtained, which demonstrated findings concerning for fungal infection. He underwent right lung thoracotomy and diaphragm biopsy and was diagnosed with invasive Mucorales infection with involvement of the right lower lung lobe, diaphragm, and liver (figure 1A). Therapy was initiated with intravenous liposomal amphotericin $\mathrm{B}$, micafungin, and posaconazole (switched to isavuconazonium sulfate after 4 days); intrathoracic amphotericin B deoxycholate and granulocyte infusions were also administered. The patient remained febrile with severe pancytopenia and severe concurrent BK virus hemorrhagic cystitis. He underwent thorascopic resection of the necrotic lung by wedge resection. A posterior right mini-thoracotomy was also performed for achievement of liver debridement. Following extensive discussion regarding risk of mortality due to underlying disease progression, extensive comorbid infection, and inability to attain effective immune reconstitution in the face of heavy leukemic burden in the marrow, the decision was made to proceed with tisagenlecleucel. Lymphodepleting chemotherapy (fludarabine (dose: $30 \mathrm{mg} / \mathrm{m}^{2} /$ day) and cytarabine (dose: $1 \mathrm{~g} / \mathrm{m}^{2} /$ day), modified due to severe lymphocytopenia and known alkylator-resistant disease) was administered over 2 days. At the time of infusion, the patient was normotensive, though still febrile and hypoxic (on supplemental nasal cannula) with three chest tubes in place following thoracotomy. The patient tolerated tisagenlecleucel infusion.

Fever frequency and intensity increased several days after infusion, consistent with CRS. The patient remained normotensive with minimal oxygen requirement. On postinfusion day 8 , tocilizumab was administered for a temperature of $42.9^{\circ} \mathrm{C}$. Fever returned 12 hours later but eventually resolved 7 days after tocilizumab administration (figure 2A). No other signs or symptoms of CRS materialized. To help decide between palliative therapy and continued care with curative intent, an early response evaluation was performed 13 days after infusion. Bone marrow obtained at that time showed acellular morphology with $0.02 \%$ leukemia by flow cytometry. Given the low leukemic burden in the bone marrow and anticipated low likelihood of recurrent CRS, attention 


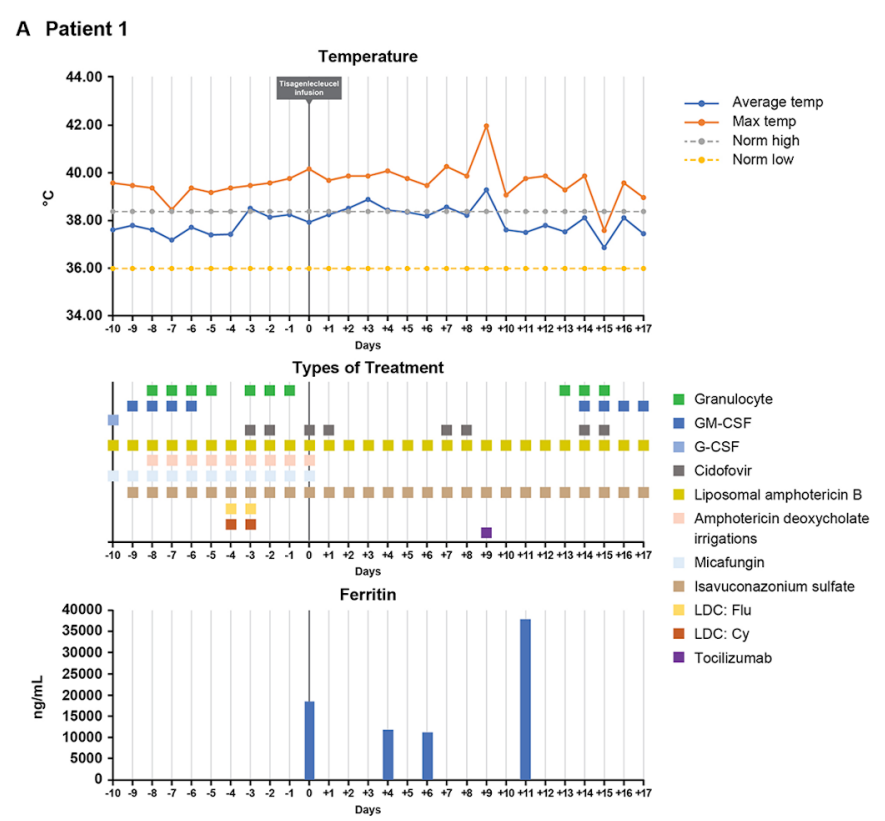

CRP

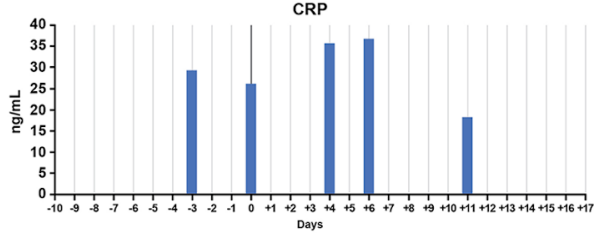

B Patient 2
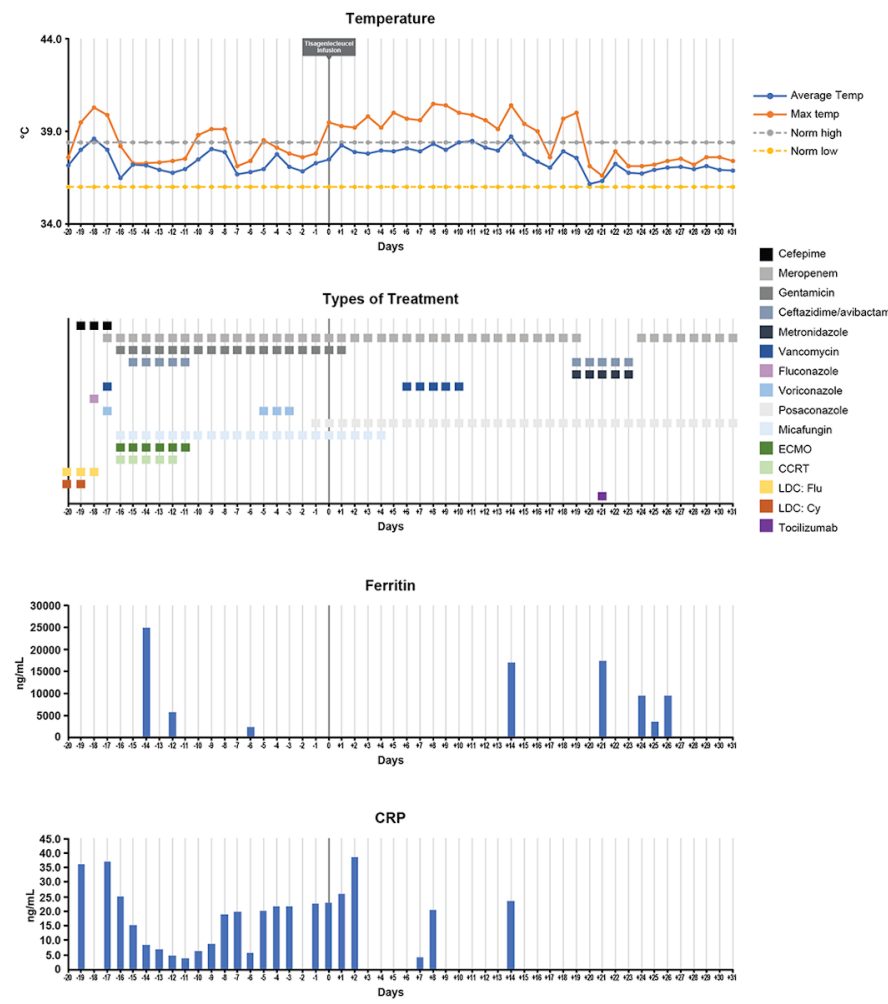

Figure 2 Temperature, treatment, ferritin levels, and CRP levels before and after tisagenlecleucel infusion. (A) Patient 1 temperature, treatment, ferritin levels, and CRP levels. (B) Patient 2 temperature, treatment, ferritin levels, and CRP levels. CRRT, continuous renal replacement therapy; CRP, C reactive protein; Cy, cyclophosphamide; ECMO, extracorporeal membrane oxygenation; Flu, fludarabine; G-CSF, granulocyte colony-stimulating factor; GM-CSF, granulocyte-macrophage colony-stimulating factor; LDC, lymphodepleting chemotherapy.

was turned to supporting hematopoiesis, which would be required to fully eradicate the invasive fungal infection. With this, granulocyte-macrophage colony-stimulating factor (GM-CSF) was initiated and granulocyte infusions were resumed. A repeat marrow was performed on day 28 postinfusion and showed a hypocellular marrow with no immunophenotypic evidence of disease. Due to persistent cytopenias and marrow hypocellularity, consideration was given to a CD34+ stem cell-selected boost from the patient's mother (stem cell donor); by day +34 post-infusion, however, the patient achieved a spontaneous rise in his absolute neutrophil count. Granulocyte infusions were discontinued, and stem cell donation was canceled. The patient was discharged from the hospital 39 days after infusion. He remained on isavuconazonium, liposomal amphotericin B, and GM-CSF at time of discharge. Fifty days after infusion, GM-CSF was discontinued (figure 3A); no significant side effects were observed due to the use of GM-CSF.

Forty-nine days after infusion, an additional thoracoscopic surgery was performed to resect residual necrotic lung; no fungal elements were detected at that time (figure 1A), and antifungal therapy was narrowed to monotherapy with isavuconazonium. The patient returned to his country of origin 76 days after infusion and remains in remission 19 months after infusion.

\section{Patient 2}

A preschool-age Hispanic female was diagnosed with preB-ALL 14 months before tisagenlecleucel infusion and was treated according to the National Mexican Protocol, initially with achievement of complete remission (see online supplemental file). She developed relapsed disease 7 months prior to tisagenlecleucel infusion. She underwent reinduction chemotherapy, blinatumomab, and focal radiation; despite these therapies, bone marrow evaluation continued to show active disease 1 year after diagnosis, at which time she was referred for tisagenlecleucel infusion.

The patient underwent leukapheresis 1 month prior to tisagenlecleucel infusion. She received bridging chemotherapy of 6-mercaptopurine and methotrexate and underwent lymphodepleting chemotherapy of fludarabine $\left(30 \mathrm{mg} / \mathrm{m}^{2} /\right.$ dose for 3 days) and cyclophosphamide $\left(500 \mathrm{mg} / \mathrm{m}^{2} /\right.$ dose for 2 days) 18 days prior to tisagenlecleucel infusion. Within 3 days of starting lymphodepleting chemotherapy, while neutropenic, she developed severe septic shock (extended spectrum betalactamase-positive and ertapenem-resistant Escherichia coli) with need for broad-spectrum antibiotics, steroids, inotropes, ventilatory support, 4 days of continuous renal replacement therapy and 5 days of venoarterial extracorporeal membrane oxygenation (VA-ECMO). 


\section{A Male, age 23 at diagnosis}

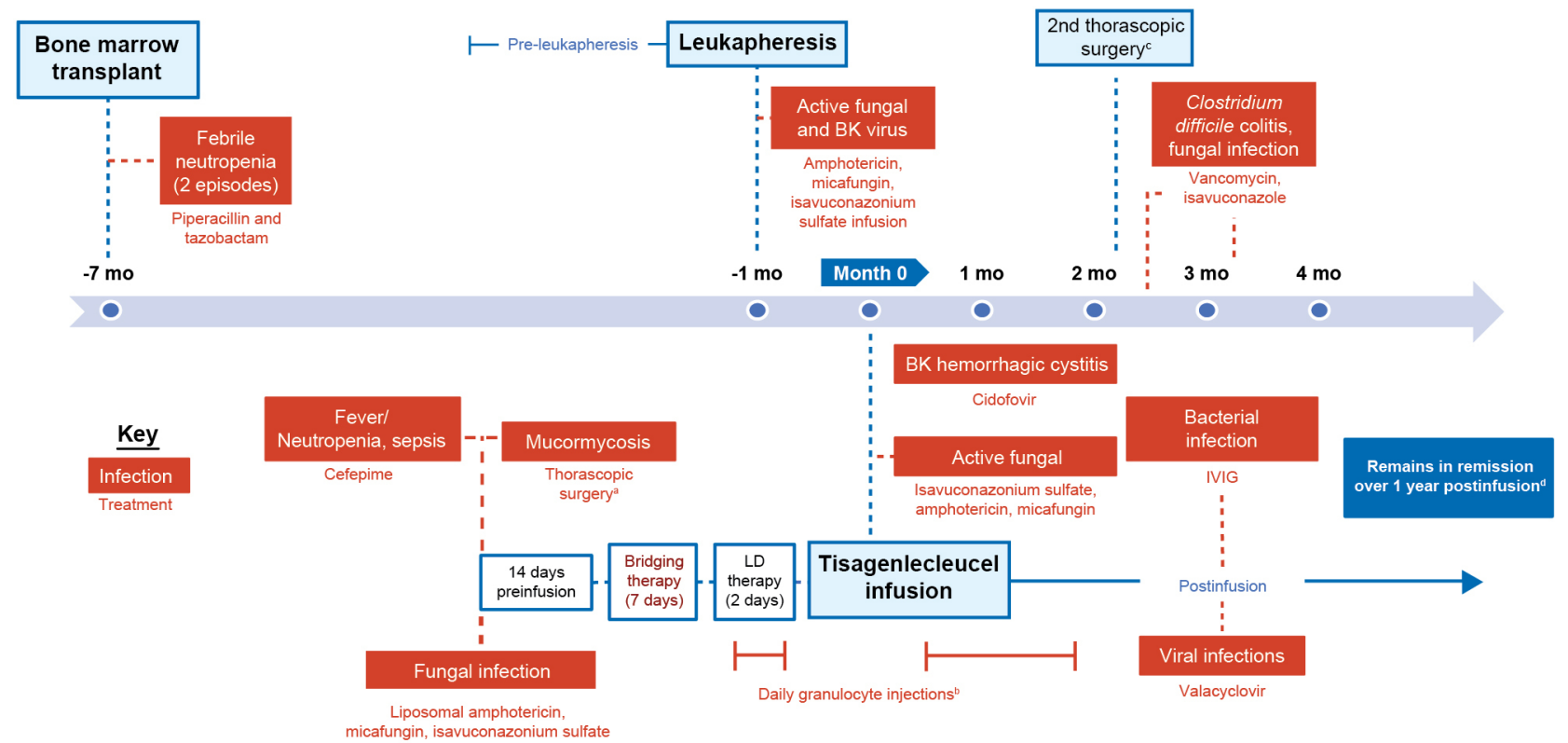

\section{B Female, preschool age at diagnosis}

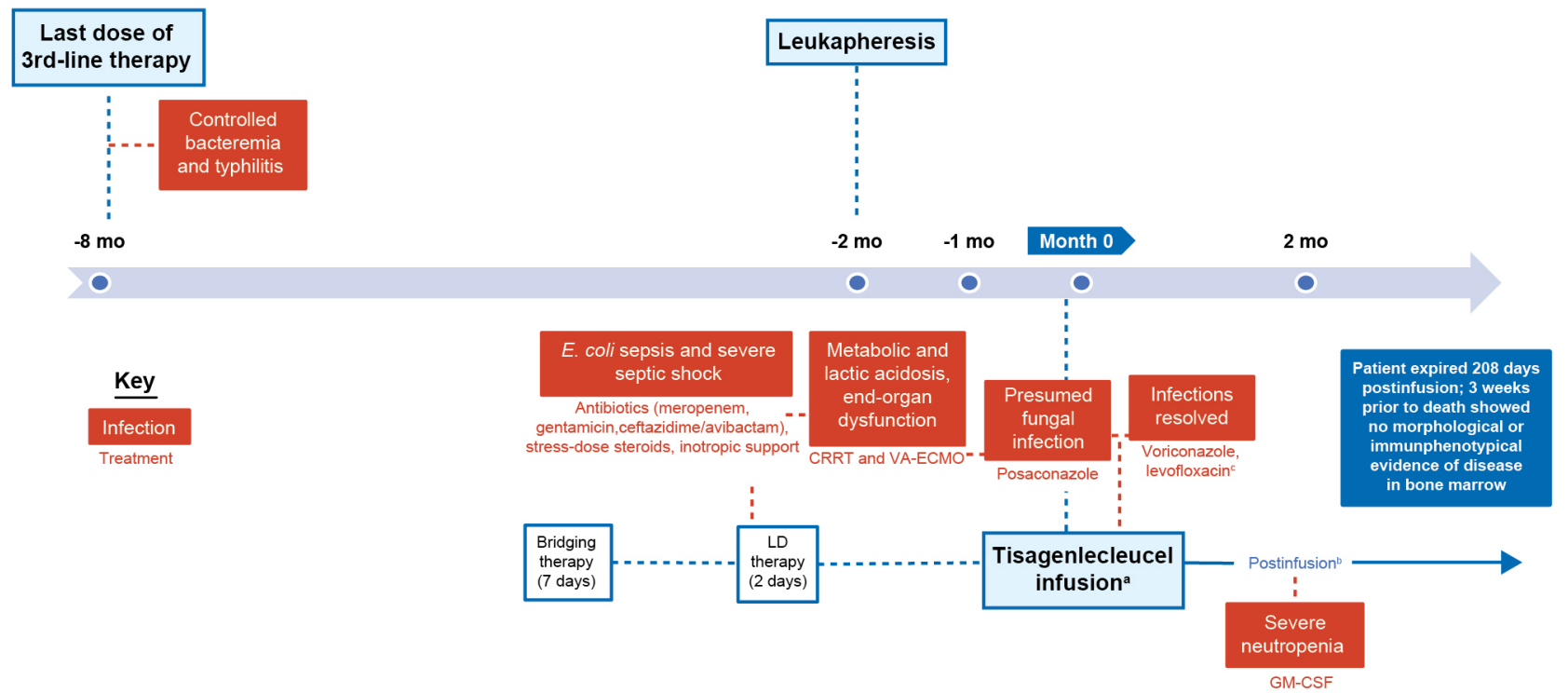

Figure 3 Timeline of infections and treatment throughout the tisagenlecleucel therapy process. (A) Timeline for patient 1. ${ }^{a}$ Day 8 of cefepime found muscle swelling and fungal elements in the liver; thus, the patient underwent surgery (right videoassisted thorascopic surgery with right lower lobe lung wedge resection, right thoracotomy, diaphragm biopsy, and right liver lobe wedge resection). ' Discontinued 2 days before infusion and restarted 14 days after until end of September 2018. 'Videoassisted thorascopic surgery in which a small portion of the right middle lung lobe was resected from the pleura and showed negative fungal elements. Lesion was not patched due to the potential of fungal growth. ${ }^{\mathrm{d}}$ Ongoing observation. (B) Timeline

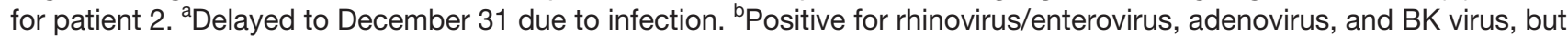
remained asymptomatic. ${ }^{\circ}$ Continued throughout blast count recovery. CRRT, continuous renal replacement therapy; GMCSF, granulocyte-macrophage colony-stimulating factor; IVIG, intravenous immunoglobulin; LD, lymphodepleting; VA-ECMO, venoarterial extracorporeal membrane oxygenation.

VA-ECMO was discontinued 11 days prior to tisagenlecleucel infusion. Shortly thereafter, the patient developed recurrent fever. A CT scan obtained for evaluation of an infectious nidus showed pulmonary opacities with tree-in-bud appearance (possible fungal etiology) and a small intra-abdominal abscess/phlegmon. Given these 
findings, she was started on posaconazole in addition to existing broad-spectrum antimicrobials (figure 2B). With consideration given to disease burden ( $11 \%$ circulating leukemic blasts on day prior to infusion), optimal antifungal/antimicrobial therapy, and stable clinical exam, the decision was made to proceed with tisagenlecleucel infusion (figure 1B). At the time of infusion, respiratory failure was resolved (without need for non-invasive ventilatory support) and the patient was hemodynamically stable. She remained on amlodipine for management of underlying hypertension, but all inotropic support was discontinued. An echocardiogram (obtained 4 days preinfusion) showed qualitatively normal right ventricular systolic function and mild global hypokinesis of the left ventricle (ejection fraction (EF) 49\%, shortening fraction (SF) 32\%).

The patient's postinfusion course was complicated by CRS (characterized by fever, cardiac dysfunction, fluid overload, and respiratory insufficiency) and extensive lymphadenopathy (figure 1B) with associated renovascular compromise. She received two doses of tocilizumab on day+21 given 12 hours apart with some clinical improvement, though ultimately with need for resumption of non-invasive ventilatory support and milrinone due to depressed left ventricular systolic function (EF $45 \%$, SF $21.5 \%$ ). Given the unclear nature of the patient's aforementioned extensive adenopathy and inability to exclude disease progression, a bone marrow biopsy and aspirate were completed on day 21 postinfusion; it revealed persistent marrow disease, though notably with numerous $\mathrm{CD} 3+\mathrm{T}$ lymphocytes surrounding visualized blast clusters (figure 4A-E). Follow-up biopsy and aspirate obtained 31 days postinfusion showed extensive fibrosis with no morphological or immunophenotypic evidence of disease (figure $4 \mathrm{~F}$ ). The patient was discharged from the hospital 53 days after tisagenlecleucel infusion. An echocardiogram obtained prior to discharge showed mildly depressed left ventricle systolic function (EF 49\%, SF 26\%) with mild left ventricular dilation. At the time of discharge (day +54 postinfusion) and on transfer home to her country of origin $($ day +58$)$, she remained on amlodipine for management of hypertension but was otherwise on no cardiac remodeling medications. She remained on granulocyte colony-stimulating factor for management of severe neutropenia and was transfusion-dependent (packed red blood cells and platelets). In the setting of severe neutropenia, levofloxacin and voriconazole were also continued for infection prophylaxis.

The patient returned to her country of origin 58 days after infusion. Unfortunately, she expired 208 days after infusion due to cardiac arrest of unclear etiology (figure 3B). An autopsy was not obtained. Her last bone marrow evaluation was completed 3 weeks prior to death and showed no morphological or immunophenotypic evidence of disease.

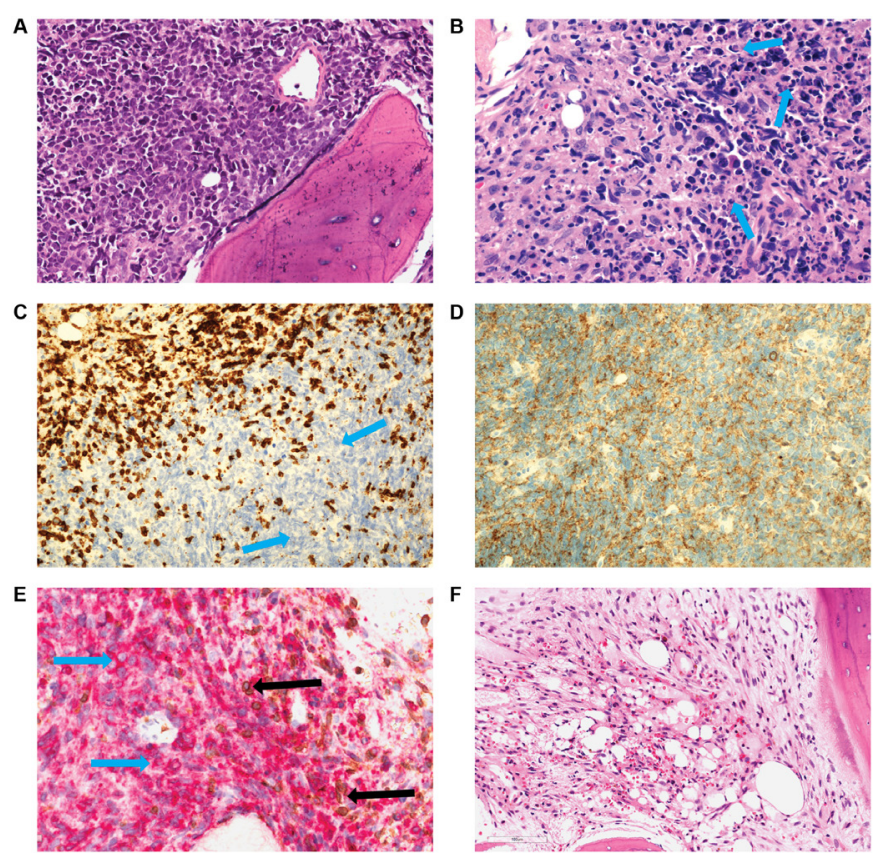

Figure 4 Bone marrow biopsy for patient 2 before and after tisagenlecleucel. (A-F) Pathology of biopsies for patient 2.

Pathology of the bone marrow at 21 days $(A-E)$ and 31 days $(F)$ after infusion. $(A, B)$ Blasts in the bone marrow exhibited karyorrhexis (blue arrows) mixed with intact peripheral cells 21 days after infusion. (C) CD3+ T cells surrounding and infiltrating leukemic blasts. Blue arrows indicate CD3negative blast cells. (D) CD19 expression on the blasts. (E) Dual CD3-CD10+ immunostaining highlight $T$ cells in brown (black arrows) and blasts in red (blue arrows). (F) Bone marrow at day 31 , depleted of hematopoietic cells.

\section{DISCUSSION}

Here, we describe two patients with heavily pretreated $\mathrm{r} / \mathrm{r}$ B-ALL who were safely infused with tisagenlecleucel despite elevated inflammatory markers, heavy leukemic burden, significant infection, and multiple comorbidities. While our center has experience with infusion of CAR-T cells dating back to 2012 and physician experience with infusions dating to 2005, tisagenlecleucel infusions are and will be the first such infusions for many centers. The complex management of patients prior to, during, and after infusion can be a source of significant concern for many providers, particularly in the presence of underlying infection, evolving disease burden, and/or other confounding variables that increase risk for severe CRS. Early recommendations to sites regarding conditions for infusion and CRS management on the pivotal multisite ELIANA trial of tisagenlecleucel were based on adult and pediatric experience at a single institution. During the trial, experienced providers were frequently consulted by those centers or providers with less experience in CAR-T cell infusion when challenged by cases for which the existing CRS management guidelines did not provide clear guidance on how to proceed (ie, a provider may inquire about tocilizumab for a patient with 5 days of persistent fever to $40^{\circ} \mathrm{C}$ without other signs or symptoms of CRS). 
Earlier in the ELIANA trial, there was significant concern that early CRS intervention might lead to diminished efficacy. Through increased patient experience in both trial and real-world settings, however, much has been learned about patient management, including management of mild to severe CRS and neurological toxicity. ${ }^{4}$ As the number of sites administering tisagenlecleucel expands and a wide range of real-world experience is obtained, it will be difficult to implement rapid changes to official guidelines. In the ELIANA trial, $43 \%$ of patients experienced infections within 8 weeks of tisagenlecleucel infusion, and $24 \%$ were severe infections (grade $3 / 4$ ). ${ }^{2}$ In a few patients, prolonged severe neutropenia led to severe (grade 3 human herpesvirus 6 encephalitis) and fatal (encephalitis and systemic mycosis) infections. Analysis of real-world data in patients with B-ALL from the Center for International Blood and Marrow Transplant Research (CIBMTR) patient registry reported a similar incidence of postinfusion infections $(42 \%)$, with one fatal bacterial infection. ${ }^{5}$ Furthermore, management of CRS with short-term tocilizumab therapy (IL-6 blockade) does not appear to increase the risk of infection ${ }^{6}$; however, it is important to consider that immune responses to invasive fungal infections could involve IL- $6^{78}$ and therefore may respond differently to tocilizumab therapy than was reported for our patient. Taken together, these clinical trial and real-world data highlight the known postinfusion infection risk in these patients; however, existing data fail to provide insight into how patients with preinfusion infections might be managed. Expanding the existing literature to further describe management of complex patients who, in some respects, challenge the current guidelines for infusion will be important for improving access to therapy for patients who lack other options for potential durable remission. Technically, the patients reported here were infused with tisagenlecleucel outside of label guidelines due to the presence of elevated inflammatory markers and active infection. ${ }^{1}$ For patient 1, CRS manifestations were limited to only fever without hypotension or progressive renal or pulmonary involvement. Response of B-ALL to tisagenlecleucel was not compromised by the treatment of CRS at this stage with tocilizumab. For patient 2, CRS was characterized by fever, third-spacing, cardiac dysfunction, and marked lymphadenopathy with secondary renal vascular compromise (suspected to be driven by CAR-T cell expansion). Cardiac dysfunction due to CRS has been observed in children undergoing CAR-T cell therapy, and risk of dysfunction may be increased in patients who have pre-existing cardiac abnormalities; persistent dysfunction remains uncommon, however. ${ }^{910}$ For patient 2 , improvement in cardiac function was observed following resolution of CRS, though with unexpected death due to cardiac arrest greater than 200 days after infusion. The etiology of this arrest remains unknown at this time.

Through experience gained treating CRS in patients who were part of the ELIANA study, we felt relatively comfortable that we could control CRS and therefore infused both patients under circumstances in which other providers might not have. Nevertheless, tisagenlecleucel infusion was only carried out after extensive counseling regarding the possibility of worst-case scenarios and with serious consideration also given to transport home for palliative measures. In both cases, significant discussions regarding potential life-threatening outcomes were had with patients, families, and medical providers prior to infusion.

\section{CONCLUSIONS}

Publication of these case studies and others may guide providers and families as they face similarly challenging circumstances in the future. In addition, they highlight the importance of clear communication with patients, families, and medical team members about potentially lethal adverse events of CAR-T cell therapy when infused in the setting of concurrent life-threatening comorbidities. While these cases represent successful infection management during CAR-T cell therapy, they should not be viewed as typical or even likely outcomes. Treatment of both patients was carried out in a setting with extensive institutional knowledge by an experienced team with the ability to customize standard protocols. Additional reporting of outcomes in patients with active infections via the CIBMTR patient registry is needed to confirm the generalizability of our approach and to better delineate benefit-risk assessment in these patients. Careful reporting on patient experiences and outcomes in the postcommercialization era of tisagenlecleucel will help to ensure safe access to potentially curative therapy for more patients.

Correction notice This article has been corrected since it first published. The provenance and peer review statement has been included.

Contributors All authors treated patients and contributed to data collection and interpretation. EMH and GDM provided data and helped write the first draft. All authors were involved in revising the manuscript, and read and approved the final version.

Funding Medical writing support was provided by Briana Dye, $\mathrm{PhD}$, of Healthcare Consultancy Group, and was funded by Novartis Pharmaceuticals Corporation.

Competing interests DEY has been an investigator on studies supported by Astellas, Chimerix, Merck, Pfizer, and Viracor-Eurofins. KJA is on the Speaker's Bureau for and receives support from Novartis Pharmaceuticals. GDM is a consultant and is on the Speaker's Bureau for Novartis Pharmaceuticals. EMH, RKG, AAA, GSM, SDS, TGF, IAA, WL, and RJH declare that they have no competing interests.

Patient consent for publication Obtained.

Ethics approval This is a retrospective case series for which institutional review board permissions were granted and results were pulled from patients' charts.

Provenance and peer review Not commissioned; externally peer reviewed.

Supplemental material This content has been supplied by the author(s). It has not been vetted by BMJ Publishing Group Limited (BMJ) and may not have been peer-reviewed. Any opinions or recommendations discussed are solely those of the author(s) and are not endorsed by BMJ. BMJ disclaims all liability and responsibility arising from any reliance placed on the content. Where the content includes any translated material, BMJ does not warrant the accuracy and reliability of the translations (including but not limited to local regulations, clinical guidelines, terminology, drug names and drug dosages), and is not responsible for any error and/or omissions arising from translation and adaptation or otherwise. 
Open access This is an open access article distributed in accordance with the Creative Commons Attribution Non Commercial (CC BY-NC 4.0) license, which permits others to distribute, remix, adapt, build upon this work non-commercially, and license their derivative works on different terms, provided the original work is properly cited, appropriate credit is given, any changes made indicated, and the use is non-commercial. See http://creativecommons.org/licenses/by-nc/4.0/.

\section{REFERENCES}

1 Novartis Pharmaceuticals Corp. Kymriah [package insert]. East Hanover, NJ: Novartis Pharmaceuticals Corp, 2018.

2 Maude SL, Laetsch TW, Buechner J, et al. Tisagenlecleucel in children and young adults with B-cell lymphoblastic leukemia. $N$ Engl J Med 2018;378:439-48.

3 Grupp SA, Maude SL, Rives S, et al. Updated analysis of the efficacy and safety of tisagenlecleucel in pediatric and young adult patients with relapsed/refractory $(r / r)$ acute lymphoblastic leukemia. Blood 2018:132:895.

4 Gardner RA, Ceppi F, Rivers J, et al. Preemptive mitigation of CD19 CAR T-cell cytokine release syndrome without attenuation of antileukemic efficacy. Blood 2019;134:2149-58.
5 Grupp S, Hu Z-H, Zhang Y, et al. Tisagenlecleucel chimeric antigen receptor (CAR) T-cell therapy for relapsed/refractory children and young adults with acute lymphoblastic leukemia (ALL): real world experience from the Center for International Blood and Marrow Transplant Research (CIBMTR) and Cellular Therapy (CT) Registry. Blood 2019;134:2619.

6 Frigault MJ, Nikiforow S, Mansour MK, et al. Tocilizumab not associated with increased infection risk after CAR T-cell therapy: implications for COVID-19? Blood 2020;136:137-9.

7 Conti HR, Gaffen SL. IL-17-mediated immunity to the opportunistic fungal pathogen Candida albicans. J Immunol 2015;195:780-8.

8 Rawlings SA, Heldt S, Prattes J, et al. Using interleukin 6 and 8 in blood and bronchoalveolar lavage fluid to predict survival in hematological malignancy patients with suspected pulmonary mold infection. Front Immunol 2019;10:1798.

9 Burstein DS, Maude S, Grupp S, et al. Cardiac profile of chimeric antigen receptor $\mathrm{T}$ cell therapy in children: a single-institution experience. Biol Blood Marrow Transplant 2018;24:1590-5.

10 Shalabi H, Sachdev V, Kulshreshtha A, et al. Impact of cytokine release syndrome on cardiac function following CD19 CAR-T cell therapy in children and young adults with hematological malignancies. J Immunother Cancer 2020;8:e001159. 


\section{Correction: Tisagenlecleucel infusion in patients with relapsed/refractory ALL and concurrent serious infection}

Hall EM, Yin DE, Goyal RK, et al. Tisagenlecleucel infusion in patients with relapsed/ refractory ALL and concurrent serious infection. J ImmunoTher Cancer 2021;9:e001225. doi: 10.1136/jitc-2020-001225

This article has been corrected since it first published. The provenance and peer review statement has been added.

Open access This is an open access article distributed in accordance with the Creative Commons Attribution Non Commercial (CC BY-NC 4.0) license, which permits others to distribute, remix, adapt, build upon this work non-commercially, and license their derivative works on different terms, provided the original work is properly cited, appropriate credit is given, any changes made indicated, and the use is non-commercial. See http://creativecommons.org/licenses/by-nc/4.0/.

(c) Author(s) (or their employer(s)) 2021. Re-use permitted under CC BY-NC. No commercial re-use. See rights and permissions. Published by BMJ.

J Immunother Cancer 2021;9:e001225corr1 . doi:10.1136/jitc-2020-001225corr1

A) Check for updates 